\title{
Corrélation entre la qualité de la réponse aux appels d'un Samu-Centre 15 et les effectifs d'assistants de régulation médicale - Étude QRAARM
}

\section{Correlation between a Samu-Center 15 response quality and medical dispatching assistants workforce - The QRAARM Study}

\author{
L. Goix · T. Petrovic $\cdot$ P.-G. Reuter $\cdot$ E. Chanzy $\cdot$ F. Linval $\cdot$ P. Wipf $\cdot$ F. Adnet $\cdot$ F. Lapostolle \\ Reçu le 18 septembre 2017; accepté le 5 mars 2018 \\ (C) SFMU et Lavoisier SAS 2018
}

Résumé Introduction : Le taux d'occupation des lignes téléphoniques d'un Samu-Centre 15 conditionne la fluidité de la gestion des appels et donc sa capacité de répondre à l'urgence. La disponibilité des assistants de régulation médicale (ARM) et des médecins régulateurs est déterminante. Nous nous sommes interrogés sur l'impact du nombre d'ARM sur la prise en charge des appels d'un Samu-Centre 15.

Méthodes : Le taux d'appels raccrochés (par l'appelant) après 15 secondes a été retenu comme critère de jugement principal. Il a été analysé en fonction de l'effectif « réel» d'ARM. L'effectif théorique d'ARM a été pris comme référence. Deux analyses ont été réalisées, la première portant sur 672 périodes d'un quart d'heure de la semaine 51 de l'année 2016 (en période d'épidémie de grippe) et la seconde sur 3624 périodes d'une heure sur les cinq premiers mois de l'année 2017.

Résultats : Sur la première période, le nombre médian d'appels raccrochés après 15 secondes était de $1(0-3)$ par quart d'heure. Il variait de $0(0-1)$ à $3(1-5)$ selon l'effectif d'ARM auquel il était parfaitement inversement corrélé $(p<0,001)$. Sur la seconde période, le nombre médian d'appels raccrochés était de 4 (1-8) par heure. Il variait

\footnotetext{
L. Goix · T. Petrovic $\cdot$ P.-G. Reuter $\cdot$ E. Chanzy $\cdot$ F. Linval

P. Wipf $\cdot$ F. Adnet $\cdot$ F. Lapostolle $(\square)$

Samu 93, UF Recherche-Enseignement-Qualité

université Paris-XIII, Sorbonne-Paris-Cité, Inserm U942,

Assistance publique-Hôpitaux de Paris,

hôpitaux universitaires de Paris-Seine-Saint-Denis,

hôpital Avicenne,

125, rue de Stalingrad, F-93009 Bobigny, France

e-mail : frederic.lapostolle@avc.aphp.fr

P.-G. Reuter

Samu 92, Assistance publique-Hôpitaux de Paris, hôpitaux universitaires de Paris, IDF Ouest,

hôpital Raymond-Poincaré,

104, boulevard Raymond-Poincaré, F-92380, Garches, France
}

de 2 (1-6) à 10 (6-16) selon l'effectif d'ARM auquel il était parfaitement inversement corrélé $(p<0,001)$.

Conclusion : Le taux d'appels raccrochés après 15 secondes était parfaitement corrélé à l'effectif (plus précisément au sous-effectif) d'ARM. Sur une année, le nombre estimé d'appels perdus ainsi était proche de 50000 ! Prendre en considération l'effectif des ARM est une nécessité absolue.

Mots clés Samu $\cdot$ Régulation · ARM $\cdot$ Effectif
Performance $\cdot$ Réponse

Abstract Introduction: The rate of occupancy of SAMUCenter 15 telephone lines determines the fluidity of the call management and therefore the capacity to respond to the emergency. The availability of medical dispatching assistants (ARMs) and emergency physician is crucial. We questioned the impact of the number of ARMs on the handling of calls in a Samu-Center 15.

Methods: The rate of on-hook calls (by the caller) after 15 seconds was used as the primary end-point. It was analyzed according to ARM's "real" workforce. The theoretical number of ARM was taken as a reference. Two analyzes were carried out, the first covering 672 periods of $1 / 4$ hour of week 51 of the year 2016 (during an influenza epidemic) and the second covering 3,624 periods of one hour out of the five first months of 2017.

Results: In the first period, the median number of on-hook calls after 15 seconds was $1(0-3)$ per $1 / 4$ hour. It varied from $0(0-1)$ to $3(1-5)$ depending on the number of ARMs to which it was perfectly inversely related $(P<0.001)$. In the second period, the median number of on-hook calls was 4 (1-8) per hour. It varied from 2 (1-6) to 10 (6-16), depending on the number of ARMs to which it was perfectly inversely related $(P<0.001)$.

Conclusion: The rate of calls hung up after 15 seconds was perfectly correlated with the number of ARMs. Over one 
year, the estimated number of lost calls was close to 50,000 ! Consideration of ARMs membership is an absolute necessity.

Keywords Emergency call - Samu - Dispatching center * Performance · Workforce

\section{Introduction}

La régulation médicale est un acte médical à part entière, réalisé au téléphone par un médecin régulateur d'un SamuCentre 15 [1]. Elle a pour but de déterminer et de déclencher une réponse médicale adaptée à chaque situation, dans les meilleurs délais. Puisqu'il s'agit de prendre en charge de façon optimale des urgences, tout retard de prise de décision est préjudiciable. Le premier des retards peut survenir en amont même de la prise en charge de l'appel par le Centre de réception et de régulation des appels (CRRA), par un délai de décroché excessif. Inévitablement, un allongement de ce délai de décroché conduit un certain nombre d'appelants à raccrocher. Il apparaît assez clairement que la charge de travail représentée par le nombre d'appels est un déterminant direct du délai de décroché et de régulation des appels. Ainsi, classiquement, le délai de régulation des appels est augmenté en période d'épidémie [2].

L'appel au Samu-Centre 15 est initialement pris en charge par un assistant de régulation médicale (ARM). Il a pour mission de localiser le patient, d'identifier sa demande et de caractériser le niveau d'urgence pour hiérarchiser les transferts d'appels au médecin régulateur. Il apparaît ainsi que l'allongement du délai de prise en charge d'un appel et subséquemment de régulation et de décision médicales pour une urgence peut aussi être imputable au centre de régulation. Le taux d'occupation des lignes téléphoniques des ARM et des médecins régulateurs conditionne la fluidité de la gestion des appels. L'inadéquation entre les ressources humaines disponibles et le flux d'appels expose donc à un allongement des délais de décroché pouvant provoquer l'interruption de la communication par l'appelant, donc la perte d'un certain nombre d'appels. C'est, dans la chaîne de prise en charge des appels d'urgence, le premier critère d'évaluation. Nous nous sommes récemment demandé si le nombre d'ARM postés au CRRA avait une influence sur les modalités de prise en charge des appels arrivant au SamuCentre 15.

\section{Méthodes}

La Seine-Saint-Denis est un département francilien totalement urbanisé et limitrophe de Paris qu'il borde au NordEst. Sa surface est de $236 \mathrm{~km}^{2}$ pour une population de
1,6 million d'habitants. Cela en fait un des départements avec l'une des plus fortes densités de population française.

La régulation médicale du Samu 93-Centre 15 a reçu, en 2016, 523489 appels distribués ayant donné lieu à l'ouverture de 232037 dossiers de régulation médicale (DRM). Un total de 43,5 équivalents temps plein (ETP) d'ARM correspondant à un effectif de 49 agents participe à l'activité du CRRA. Le nombre de médecins urgentistes en poste à la régulation varie de trois (le jour) à deux (la nuit et le week-end) pour un effectif de 47 médecins. Le nombre de médecins généralistes affectés en poste à la salle de régulation est (invariablement) de deux pour un effectif de 42 médecins.

L'effectif d'ARM et sa répartition horaire ont été définis dans la perspective d'obtenir une adéquation entre les besoins exprimés par le service et les ressources humaines de l'établissement dans l'objectif de se rapprocher des recommandations professionnelles de 7,2 appels par heure [3]. Il a ensuite été ajusté, empiriquement, à l'évolution de l'activité du service. Les ARM sont répartis en cinq vacations d'horaires différents, de 7 heures 36 minutes pour les équipes diurnes et de dix heures pour l'équipe de nuit. Le nombre d'ARM présents en salle de régulation varie de quatre à sept selon la tranche horaire. Les périodes de travail des ARM font l'objet d'un « chevauchement » afin de permettre la réalisation d'une transmission. Ce chevauchement peut générer des effectifs d'ARM transitoirement supérieurs à la valeur théorique. Le nombre réel d'ARM en poste en salle de régulation est aussi, parfois, inférieur au nombre théorique. C'est cette variable que nous avons souhaité analyser.

L'activité quotidienne d'un Samu-Centre 15 peut être évaluée par le nombre d'appels reçus et par le nombre de DRM. Ils correspondent aux appels ayant donné lieu à la création d'un dossier médical et donc à une régulation médicale. Nous avons précédemment montré que le nombre quotidien de DRM était un marqueur particulièrement sensible d'un événement sanitaire [4].

La qualité de la prise en charge des appels a été évaluée à partir du nombre d'appels raccrochés après 15 secondes (appels interrompus par l'appelant). Cette borne est couramment retenue en considérant que le raccroché avant la $15^{\mathrm{e}}$ seconde correspond, la plupart du temps, à une erreur de composition de numéro de téléphone. Dans ce cas, l'appelant se rend rapidement compte de son erreur en entendant la bande d'accueil du Samu [3]. L'analyse de ces deux variables (nombre d'ARM et d'appels raccrochés) a été réalisée quart d'heure par quart d'heure. La valeur théorique de l'effectif d'ARM a été prise comme référence.

Notre analyse a porté sur une période (fin d'année 2016) au cours de laquelle était rapportée une tension dans la prise en charge des appels en raison d'une activité intense (épidémie de grippe) et d'un effectif d'ARM opérationnel parfois 
incomplet. Afin de consolider nos résultats et de nous affranchir des potentiels biais liés à l'analyse d'une période tendue, une seconde analyse a été réalisée sur une période étendue de cinq mois (à partir de janvier 2017), avec le même critère de jugement, par tranches d'une heure. Cette seconde analyse a été renforcée par l'analyse du taux d'appels décrochés en 60 secondes (QS60s).

Une analyse supplémentaire a été conduite afin d'évaluer l'impact de la « pause déjeuner »- qui peut aussi générer « artificiellement» des réductions ponctuelles d'effectifs ARM. La période de $12 \mathrm{~h} 00$ à $14 \mathrm{~h} 00$ a été arbitrairement retenue comme période de la "pause déjeuner ». L'activité pendant cette période a été comparée à celle des autres périodes.

\section{Analyse statistique}

Les périodes étudiées ont été séparées en fonction de la différence entre l'effectif théorique et l'effectif réel d'ARM logués au logiciel métier. Pour chaque tranche d'effectifs, le taux médian d'appels raccrochés au-delà de 15 secondes a été calculé. Les variables quantitatives (nombre d'appels raccrochés après 15 secondes) ont été comparées tranche à tranche par un test de Mann-Whitney et les variables qualitatives (taux d'appels décrochés après 60 secondes) par un test de $\mathrm{Chi}^{2}$. Une valeur de $p$ inférieur à 0,05 a été retenue comme significative.

Les variables sont exprimées en nombre (pourcentage) et médiane [25-75\% interquartile].

\section{Résultats}

L'analyse initiale a porté sur 672 périodes d'un quart d'heure de la semaine 51 de l'année 2016. Sur la période étudiée, le nombre d'appels reçus était de 10702 , correspondant à une médiane quotidienne de 1524 (1502-1 550). Le nombre de DRM était de 5 513, correspondant à une médiane quotidienne de 790 (751-819). L'effectif d'ARM était conforme à la théorie dans $197(29 \%)$ périodes. Il était supérieur à la théorie dans $51(8 \%)$ périodes. Il était inférieur à la théorie dans $424(73 \%)$ périodes. Le nombre médian d'appels raccrochés après 15 secondes était de $1(0-3)$. Il variait de $0(0-1)$ à $3(1-5)$ selon l'effectif d'ARM. Le nombre médian d'appels raccrochés après 15 secondes était proportionnel à l'effectif d'ARM lorsque ce dernier était inférieur ou égal à l'effectif théorique ; $p$ inférieur à 0,001 pour toutes les comparaisons (Fig. 1).

La seconde analyse a porté sur 3624 périodes d'une heure. Les résultats n'étaient pas disponibles pour sept périodes $(0,002 \%)$ en raison d'une délocalisation de la régulation du Samu. Le nombre total d'appels reçus sur la période étudiée était de 271 997. Le nombre de DRM sur la période était
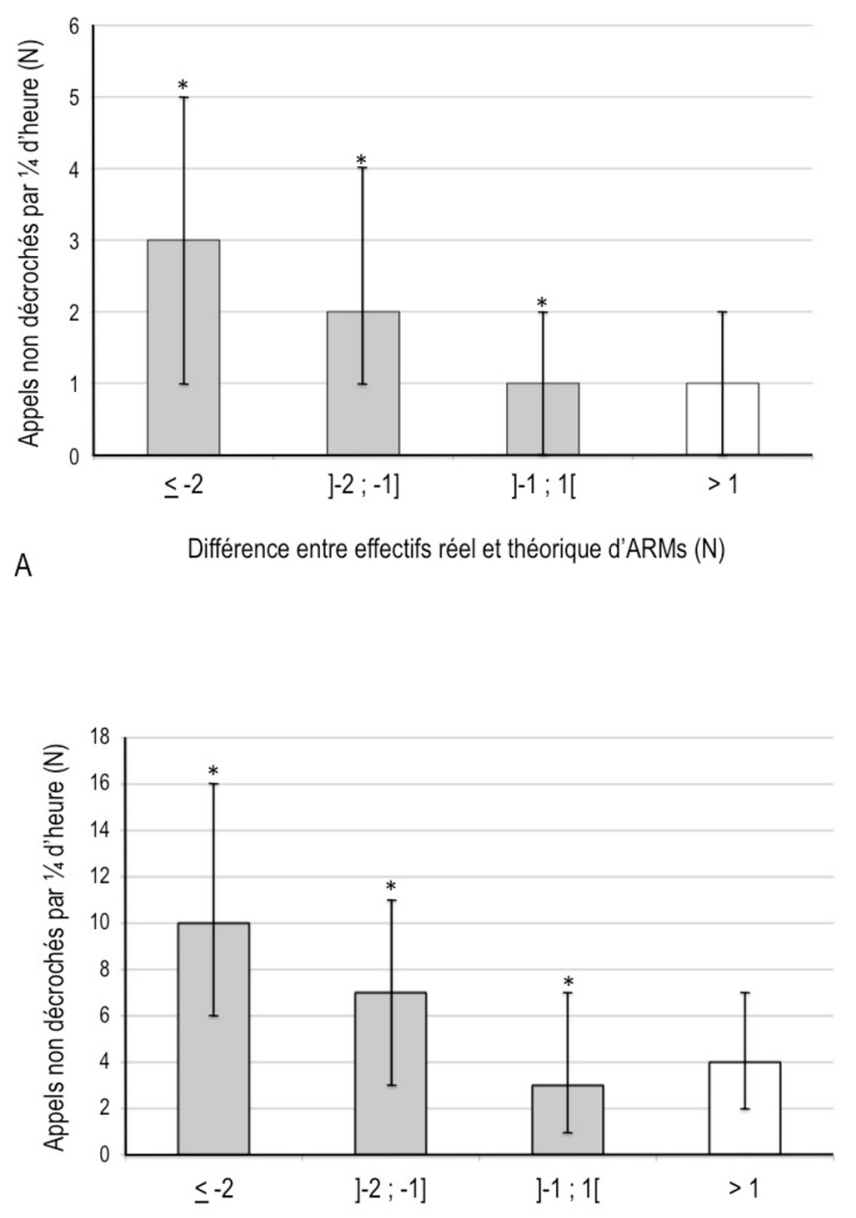

B

Différence entre effectifs réel et théorique d'ARMs $(\mathrm{N})$

Fig. 1 Taux médians d'appels raccrochés après 15 secondes (et interquartiles) en fonction de la différence entre effectifs réel et théorique d'ARM. Analyses par périodes d'un quart d'heure (A) $[n=672]$ et par périodes d'une heure (B) $[n=3624] .{ }^{*} p<$ 0,001 pour toutes les comparaisons. Les histogrammes de droite correspondent aux périodes de « sureffectif»

de 91 445, correspondant à une médiane quotidienne de 586 (541-649). Le nombre total d'appels raccrochés après 15 secondes était de 20 548. Le nombre médian horaire d'appels raccrochés était de 4 (1-8), et le nombre médian quotidien d'appels raccrochés était de 120 (100-156). Il variait de 2 (1-6) lorsque l'effectif était celui attendu à 10 (6-16), lorsque l'effectif était déficitaire d'au moins deux ARM. Le nombre médian d'appels raccrochés après 15 secondes était proportionnel à l'effectif d'ARM lorsque ce dernier était inférieur ou égal à l'effectif théorique ; $p$ inférieur à 0,001 pour toutes les comparaisons (Fig. 1).

Le nombre médian d'appels décrochés en 60 secondes était proportionnel à l'effectif d'ARM lorsque ce dernier était inférieur ou égal à l'effectif théorique ; $p$ inférieur à 0,001 pour toutes les comparaisons (Fig. 2). 


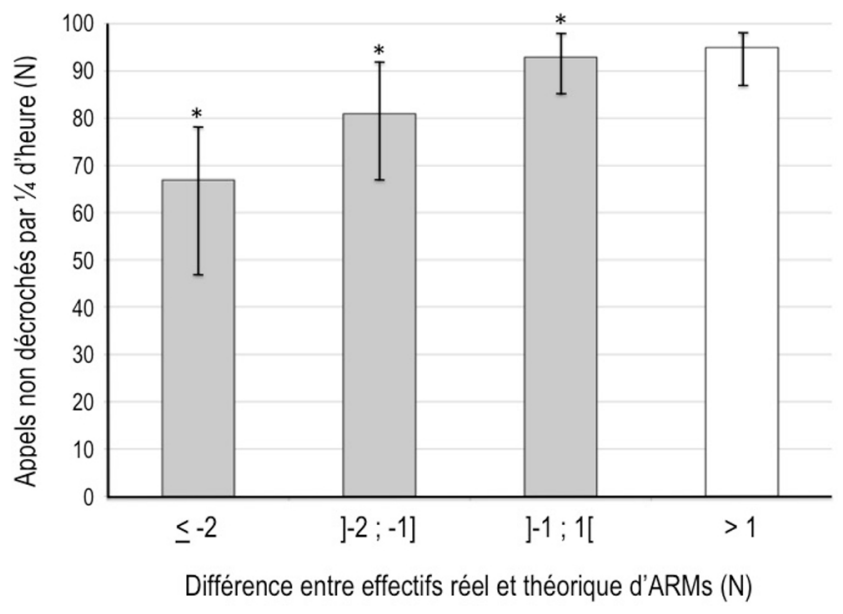

Fig. 2 Taux médians d'appels décrochés en 60 secondes (et interquartiles) en fonction de la différence entre effectifs réel et théorique d'ARM. Analyse par périodes d'une heure $(n=3$ 624). $* p<0,001$ pour toutes les comparaisons. Les histogrammes de droite correspondent aux périodes de « sureffectif»

Le taux médian horaire d'appels raccrochés après 15 secondes pendant la « pause déjeuner » était significativement plus élevé que pendant les autres tranches horaires : $9(6-13)$ vs $4(1-8)$ minutes ; $p$ inférieur à 0,001 .

\section{Discussion}

L'effectif d'ARM du service était inférieur à l'effectif théorique les trois quarts du temps. Le taux d'appels raccrochés après 15 secondes était parfaitement corrélé à l'effectif d'ARM. Sur une année, le nombre d'appels raccrochés après 15 secondes - constituant donc potentiellement des appels urgents perdus - dépasse les 50000 (résultat non présenté : 54226 abandons au-delà de 15 secondes d'attente pour l'année 2016) ! Ainsi, il apparaît que des éléments internes peuvent, avec la même intensité que des événements extérieurs, participer à diminuer la qualité de la réponse d'un SamuCentre 15.

Divers phénomènes ont été rapportés comme pouvant modifier l'activité d'un Samu-Centre 15. Il s'agit essentiellement d'événements sanitaires, comme les épidémies (grippe, gastroentérite, etc.) ou une canicule, mais pas toujours [4]. Parfois, les causes en sont toutes autres, comme des manifestations sportives ou d'autres événements $[5,6]$. Cette modification de l'activité du Samu-Centre 15 influe évidemment sur la qualité de la prestation proposée. La surcharge des Samu-Centre 15 expose certainement, comme celle des services d'urgences intrahospitaliers, à un allongement des délais de prise en charge, à une dégradation de la prise en charge et certainement à la « perte » de patients [7]. Malheureusement, cet impact des conditions extérieures sur l'activité d'un Samu-Centre 15 et ses conséquences potentielles sur la qualité des soins prodigués est peu documenté. Le nombre d'appels reçus peut augmenter, les alertes être multipliées. Pour autant, cela ne permet pas toujours de réduire les délais [8].

Nous rapportons ici que des facteurs internes peuvent aussi contribuer à dégrader la performance de manière très significative. Cet impact est majeur. In fine, évalué sur une année, le nombre d'appels perdus est considérable. Il représente l'équivalent d'un cinquième des DRM traités par le Samu 93. Certes, ces appels ne correspondaient certainement pas tous à des patients en situation d'urgence et n'auraient certainement pas tous donné lieu à la création d'un DRM. Néanmoins, ce résultat illustre le taux élevé d'appels potentiellement urgents non pris en charge. Si l'effectif médical est souvent analysé, il apparaît ici qu'il ne doit pas être le seul à considérer. L'effectif des ARM est au moins aussi important. Il est notoire que l'analyse focalisée, réalisée quart par quart d'heure sur une période considérée comme tendue (médiane d'un appel perdu par quart d'heure), était parfaitement reproduite dans la seconde période avec une médiane de quatre appels perdus par heure. Ce type d'étude doit permettre d'affiner les recommandations professionnelles. Cette préoccupation est largement étudiée dans d'autres systèmes $[9,10]$.

Par ailleurs, la réglementation française, à l'inverse de celle d'autres pays, ne fait pas référence au délai de réponse des centres publics d'appels [11]. Seule la charte Marianne fait état d'un engagement de tous les services publics à répondre à tout appel téléphonique en moins de cinq sonneries [12]. Des indicateurs de qualité pour les SamuCentre 15 ont été promus par les recommandations professionnelles [3]. Il s'agit, par exemple, du taux d'accueil, c'està-dire du nombre d'appels abandonnés au-delà de 15 secondes sur le nombre d'appels présentés. L'objectif professionnel est que ce taux soit inférieur à $1 \%$. Ce critère est proche de celui que nous avons utilisé. Pourtant, il nous semble qu'évalué sous forme d'un rapport il rend insuffisamment compte du volume global d'appels concernés, ici supérieur à 50000 par an. Ce critère est le premier à considérer, car il conditionne l'accès même au système de soins. L'analyse de l'aval (QS60s) montre, sans surprise des résultats comparables, les déterminants de la performance étant communs. D'autres pays ont des réglementations nettement plus contraignantes. Ainsi, le district de Columbia (États-Unis) fixe, pour les appels au 911 , une cible de $90 \%$ de réponses en moins de dix secondes [13].

Notons enfin que, de façon paradoxale, les périodes de « sureffectif » étaient aussi associées à une relative diminution de la performance. Cela pourrait s'expliquer par le fait que ces périodes correspondaient aux temps de chevauchement et de transmission entre les équipes ainsi que, dans une moindre mesure, à des temps d'encadrement des nouveaux 
ARM en phase d'autonomisation. Il s'en suivrait une moindre disponibilité des ARM pour décrocher. Des mesures correctives spécifiques doivent être trouvées. Ainsi, l'impact de l'effectif d'ARM sur la réponse du CRRA étant avéré, les conséquences de la « pause déjeuner» constituent une cible privilégiée car corrigible. Dans ce cadre, nous nous interrogeons sur le rôle d'un superviseur qui aurait entre autres missions de fluidifier la prise d'appels en optimisant en continu le ratio entre effectif d'ARM et flux d'appels. Le cadre réglementaire et le mode de financement de cette activité existent $[14,15]$. Certains CRRA en sont déjà dotés. Une meilleure organisation doit aussi concourir à prévenir les risques de dégradation de la santé physique et mentale des agents et à maîtriser l'absentéisme [16]. Ce dernier point est crucial. L'absentéisme des téléopérateurs en centre d'appels est classiquement élevé, de l'ordre 15 à $25 \%$ [17]. L'absentéisme des ARM de notre service est de $10 \%$ environ quand celui des agents de l'Assistance publique-Hôpitaux de Paris (AP-HP) est de $6 \%$.

La principale limite à cette analyse est que - par définition - le motif de ces appels raccrochés demeure inconnu. Il est probable que tous ne relevaient pas d'une réelle urgence. Néanmoins, un taux réduit d'appels non décrochés est universellement considéré comme un critère de performance. Enfin, la transposition de nos résultats à d'autres centres n'est pas nécessairement naturelle. Il est vraisemblable que le niveau global quotidien d'activités module la relation entre le nombre d'ARM et le nombre d'appels perdus.

\section{Conclusion}

Le taux d'appels raccrochés après 15 secondes était très fortement corrélé à l'effectif — ou plus précisément au souseffectif - d'ARM. Sur une année, le nombre d'appels ainsi perdus pourrait dépasser 50000 ! Prendre en considération l'effectif des ARM dans l'organisation d'un SamuCentre 15 s'affiche comme une absolue nécessité.

Liens d'intérêts : les auteurs déclarent ne pas avoir de lien d'intérêt.

\section{Références}

1. Haute Autorité de santé - HAS (2011) Modalités de prise en charge d'un appel de demande de soins non programmés dans le cadre de la régulation médicale. https://www.has-sante.fr/portail/upload/docs/application/pdf/2011-10/reco2clics_regulation_ medicale.pdf (Dernier accès le 7 août 2017)

2. Anonyme (2017) Grippe : les urgences sont « aux limites de leurs capacités ». Libération. http://www.liberation.fr/france/2017/01/11/ grippe-les-urgences-sont-aux-limites-de-leurs-capacites_1540609 (Dernier accès le 2 mars 2018)

3. Samu-Urgences de France (SUdF), Société française de médecine d'urgence (SFMU) (2015) Samu-Centre 15 : Référentiel et guide d'évaluation. http://www.samu-urgences-de-france.fr/medias/files/ 155/802/sfmu-sudf_referentiel_samu_2015.pdf (Dernier accès le 7 août 2017)

4. Lapostolle F, Fleury M, Crocheton N, et al (2005) Determination of early markers of a sanitary event. The example of the heat wave of August 2003 at the Samu 93-Centre 15 in France. Presse Med 34:199-202

5. Lapostolle F, Le Toumelin P, Fleury M, et al (1999) Influence de la retransmission télévisée des matchs de la Coupe du monde de football sur l'activité du Samu 93-Centre 15. JEUR 12:105-9

6. Reuter PG, Orsini C, Grave M, et al (2018) Impact of a general practitioners' strike and of terrorist attacks on a call centre. Eur J Emerg Med in press

7. Wilson BJ, Zimmerman D, Applebaum KG, et al (2013) Patients who leave before being seen in an urgent care setting. Eur $\mathrm{J}$ Emerg Med 20:420-4

8. Stickles JL, Kempema JM, Brown LH (2018) Effect of mobile phone proliferation on crash notification times and fatality rates. Am J Emerg Med 36:24-26

9. Rutkow L, Vernick JS, Thompson CB, et al (2014) Emergency preparedness law and willingness to respond in the EMS workforce. Prehosp Disaster Med 29:358-63

10. Barnett DJ, Balicer RD, Thompson CB, et al (2009) Assessment of local public health workers' willingness to respond to pandemic influenza through application of the extended parallel process model. PloS One 4:e6365

11. Office of unified communications FY2017 (2017) 2017 Key performance indicators. https:/oca.dc.gov/sites/default/files/dc/sites/oca/ publication/attachments/OUC17.pdf (Dernier accès le 10 novembre 2017)

12. Gouvernement du Québec. Loi sur la sécurité civile. Règlement sur les normes, les spécifications et les critères de qualité applicables au centre 911 et à certains centres secondaires d'appels d'urgences. www.legisquebec.gouv.qc.ca/fr/ShowDoc/cr/S-2.3,\%20r. $\% 202 /$ (Dernier accès le 2 janvier 2018)

13. Service du Premier ministre, secrétariat général pour la Modernisation de l'Action publique (2013) Référentiel Marianne V3 juin 2013. www.modernisation.gouv.fr/sites/default/.../referentiel_marianne_vf_juin_2013.pdf (Dernier accès le 7 août 2017)

14. République française, ministre de l'Emploi, de la Cohésion sociale et du Logement (2007) Arrêté du 27 avril 2007 portant création du titre professionnel de superviseur en relation client à distance. Journal officiel de la République française 2007 version consolidé de février 2016 (Dernier accès le 2 janvier 2018)

15. Direction générale de l'offre de soins (DGOS) (2014) Groupe technique - Financement des structures de médecine d'urgence. http:// www.samu-de-france.fr/fr/ressources pro/exercice pro/exercice pro_actu/front/afficher/?id_actu=787 (Dernier accès le 7 août 2017)

16. Acker D, Bensadon AC, Legrand P, et al (2012) Management et efficience hospitalière : une évaluation des déterminants. Inspection générale des affaires sociales. http://www.igas.gouv.fr/spip. php?article280 (Dernier accès le 7 août 2017)

17. Deriot G (2009-2010). Rapport d'information au sénat $n^{\circ} 642$ fait au nom de la Mission d'information sur le mal-être au travail et de la commission des affaires sociales, déposé le 7 juillet 2010 . http://www.senat.fr/rap/r09-642-1/r09-642-1.html (Dernier accès le 07 août 2017) 